\title{
Atractylenolide III predisposes miR-195-5p/FGFR1 signaling axis to exert tumor-suppressive functions in liver cancer
}

\section{Langqing Sheng ( $\square$ xiangxiang_0102@163.com )}

Xiangya Hospital Central South University

Jiarong Li

Xiangya Hospital Central South University

Nianfeng Li

Xiangya Hospital Central South University

Liansheng Gong

Xiangya Hospital Central South University

Ling Liu

Xiangya Hospital Central South University

Qi Zhang

Xiangya Hospital Central South University

Xiaoli Li

Xiangya Hospital Central South University

Hui Luo

Xiangya Hospital Central South University

Zeguo Chen

Xiangya Hospital Central South University

\section{Research}

Keywords: Atractylenolide III, hepatocellular carcinoma, FGFR1, apoptosis, migration

Posted Date: August 12th, 2020

DOI: https://doi.org/10.21203/rs.3.rs-56832/v1

License: (c) (1) This work is licensed under a Creative Commons Attribution 4.0 International License.

Read Full License 


\section{Abstract}

Background: Antineoplastic activity of atractylenolide III (ATL) has been reported in several malignant tumors. However, its activity has not been completely clarified in hepatocellular carcinoma (HCC). Herein, anti-cancer effects and underlying molecular mechanisms of ATL were investigated in HCC cells in vitro.

Methods: Cell viability was evaluated by CCK-8 assay. Cell migration and invasion were evaluated using the transwell assay. TUNEL staining was performed to evaluate cell apoptosis. Protein expression was measured by western blotting analysis. On-line database TargetScan and luciferase reporter gene analysis were performed to validate FGFR1 as a target of miR-195-5p.

Results: HepG2 and SMMC7721 cell growth, migration and invasion were inhibited by ATL treatment in a dose-dependent pattern. ATL treatment induced apoptosis of HepG2 and SMMC7721 cells. Intriguingly, ATL treatment unexpectedly inhibited FGFR1 protein expression in HepG2 and SMMC7721 cells. Knockdown of FGFR1 inhibited proliferation, migration and invasion, and evoked apoptosis of HepG2 and SMMC7721 cells. We also found that ATL treatment could increase the expression of miR-195-5p, which as a post-transcriptional targeted FGFR1. In HCC tissues, miR-195-5p expression is negatively correlated with FGFR1. Furthermore, the anti-proliferative and pro-apoptotic roles of miR-195-5p were neutralized by overexpressed FGFR1 in HCC cells.

Conclusion: ATL effectively repressed growth and induced apoptosis of human HCC cells through the upregulation of miR-195-5p to down-regulate FGFR1 expression.

\section{Introduction}

Hepatocellular carcinoma (HCC) is the most common cause of global cancer-associated mortality with a 1-year survival rate of less than $50 \%$ [1]. Patients with advanced HCC have a poor prognosis, meanwhile recurrence and metastasis are often detected even after curative surgery [2]. Currently, palliative chemotherapy, including doxorubicin, mitomycin, cisplatin and 5-fluorouracil, is the main method for treatment and improves survival among patients with HCC [3-5]. However, nearly all patients develop into resistance to chemotherapeutic agents $[6,7]$. Thus, new treatment strategies are significant for improving therapeutic effects for patients with HCC.

Atractylenolide III (ATL) is a sesquiterpenoid and is the major active ingredient of Atractylodes macrocephala Koidz [8], and is also found in a range of other medical herbs, such as Codonopsis pilosula, Chloranthus henryi Hemsl and Atractylodes lancea. ATL is known to possess anti-inflammatory and anti-cancer properties $[9,10]$. Recently, ATL promotes human lung carcinoma A549 cells apoptosis by targeting the mitochondria-mediated death signaling pathway [9]. Hence, ATL is believed to be promising for the treatment of GC. 
The molecular mechanisms evoked in response to ATL have been investigated in cell culture and animal models $[8,11]$. Increasing data have been indicated that fibroblast growth factor receptor1 (FGFR1)/extracellular signal-regulated kinase (ERK) signaling cascade has been involved in the carcinogenesis and maintenance of common cancers [12-14]. Elevated levels of FGFR1 have been observed in various types of human cancer, including breast cancer [15], non-small cell lung cancer [16] and gastric cancer [17]. The knockdown of FGFR1 inhibits the growth of MKN-45 cells [18]. Several groups have reported that FGFR1/ERK is highly expressed in HCC, suggesting that FGFR1/ERK may serve as a potential therapeutic target for cancer therapy $[12,19,20]$. Based on FGFR1 and ERK critical roles in cancer progression, we hypothesized that FGFR1/ERK signaling cascades may be involved in anti-tumor mechanisms of ATL in HCC cell lines.

microRNAs (miRs) as non-coding RNAs contributes to post-transcriptional repression that is involved in a variety of physiological and pathological processes, including carcinogenesis [21]. Numerous miRNAs as the tumor suppressor or oncogenes have been reported in hepatic tumorigenesis [22]. A comprehensive analysis of 26 published datasets reveals that a miRNA integrated-signature of 13 miRNAs is differentially expressed in HCC tissues [22]. Among these miRNAs, miR-195-5p as a post-transcriptional regulator of FGFR1 is selected out for molecular mechanism study. miR-195-5p as a tumor suppressor is validated in several cancer subtypes, including prostate cancer [23], bladder cancer [24] and breast cancer [25]. In the present study, we aimed to investigate whether ATL induced growth inhibition and apoptosis in HCC cells by targeting miR-195-5p/FGFR1 signaling pathway.

\section{Materials And Methods Sample selection}

Human HCC tissues and adjacent para-carcinoma tissues were collected from fifty-seven HCC patients undergoing surgical operations at the Xiangya Hospital of Central South University. The clinical research was approved by the Ethics Committee of the Xiangya Hospital of Central South University. Informed consent forms were approved by all of the patients.

\section{Cell culture and drugs.}


Human HCC cell lines (MHCC97H, HepG2, Huh7 and SMMC7721) were purchased from the Institute of Biochemistry and Cell Biology of the Chinese Academy of Sciences (Shanghai, China). Cells were maintained in DMEM with $10 \%$ fetal bovine serum, $100 \mathrm{U} / \mathrm{ml}$ penicillin and $100 \mu \mathrm{g} / \mathrm{ml}$ streptomycin in a humidified incubator (Thermo, USA), $5 \% \mathrm{CO}_{2}, 95 \%$ air atmosphere. ATL was purchased from SigmaAldrich (cat. no. A2987; Merck Millipore, Darmstadt, Germany). The molecular structure of Atractylenolide III was shown in Figure $1 \mathrm{~A}$

\section{Cell transfection and plasmid constructs.}

Small interfering RNA (siRNA) was constructed to specifically silence FGFR1. siRNAs were synthesized by GenePharma (Shanghai, China). Target site in human gene encoding FGFR1 was as following: siRNAFGFR1 sense strand, 5'-GCTTCTTTCCAGCCTCTTT-3', the siRNA-FGFR1 antisense strand, 5'AAAGAGGCTGGAAAGAAGC-3'; siRNA-NC sense strand, 5'-AACTCCGGTCGAGGAGGAC-3', the siRNA-NC antisense strand, 5'-GTCCTCCCTCGACCGGAGTT-3'. siRNA-FGFR1 and siRNA-NC were transfected into HepG2 and SMMC7721 cells using Lipofectamine ${ }^{\text {TM }} 2000$ (Invitrogen, CA, USA). miR-195-5p mimics (5'UAGCAGCACAGAAAUAUUGGC-3') and scramble sequence (5'-UCAUGUAGGUAAGUGCGACGA-3') were synthesized by RiboBio (Guangzhou, China) and transfected with into HepG2 and SMMC7721 cells with a final concentration of $100 \mathrm{nM}$ using Lipofectamine 2000 (Invitrogen) for $48 \mathrm{~h}$ at $37^{\circ} \mathrm{C}$ according to the manufacturer's protocol. human FGFR1 plasmids without 3'-UTR, which did not contain the conserved complementary sequence binding with miR-195-5p, were purchased from GeneCopoeia, Inc. (Rockville, MD, USA). An empty plasmid served as negative control (vector-Con). FGFR1 overexpressed plasmids and vector-Con were transfected using Lipofectamine 2000 for $48 \mathrm{~h}$ at $37^{\circ} \mathrm{C}$, according to the manufacturer's protocols.

\section{Luciferase reporter assay.}

FGFR1 wild-type (WT) or mutant-type (Mut) 3'-UTR was inserted into the multiple cloning sites of the luciferase-expressing pMIR-REPORT vector (Ambion). For the luciferase assay, HepG2 and SMMC7721 cells containing the WT or Mut 3'-UTR of FGFR1 $(0.5 \mu \mathrm{g})$ were co-transfected with miR-Con or miR-195-5p mimics using Lipofectamine 2000. The luciferase activity was measured using a luciferase reporter assay kit (Promega Corporation, Madison, WI, USA).

\section{Cell viability detection by CCK-8.}


The CCK-8 assay (Dojindo Japan) was performed as previously described [12], human HCC cells $\left(1 \times 10^{4}\right)$ were seeded in the 96 -well plate and incubated with $\operatorname{ATL}(0,10,100$ or $500 \mu \mathrm{M})$ for 24 hours, 48 hours and 72 hours. Absorbance was recorded at $450 \mathrm{~nm}$ using Elx800 Reader (Bio-Tek Instruments Inc., Winooski, VT, USA).

\section{Migration and invasion assays.}

Cells $\left(2 \times 10^{4}\right)$ migration was analyzed using the transwell chamber $(8 \mu$ pore size; Corning Incorporated, Corning, NY, USA) without the Matrigel matrix. Cells $\left(2 \times 10^{4}\right)$ were seeded into the upper chamber precoated with Matrigel matrix (BD Biosciences) for invasion analysis. After incubation for $24 \mathrm{~h}$, cells in the down chamber were stained with $0.1 \%$ crystal violet (Beyotime) and photographed by an inverted fluorescence microscope (Leica Microsystems $\mathrm{GmbH}$, Wetzlar, Germany).

\section{TUNEL assay.}

After HepG2 and SMMC7721 cultured with different conditions, cells were collected and fixed on the glass slide. TUNEL (Beyotime Institute of Biotechnology, Haimen, China) assay was performed according to the manufacturer's instructions. TUNEL positive cells were mounted under a fluorescence microscope (Olympus, Japan).

\section{RT-qPCR.}

Total RNA was extracted using TRIzol reagent (Invitrogen; Thermo Fisher Scientific, Inc., Waltham, MA, USA). TaqMan ${ }^{\circledR}$ RT kit (Applied Biosystems; Thermo Fisher Scientific, Inc.) and TaqMan ${ }^{\circledR}$ MicroRNA assay (Applied Biosystems; Thermo Fisher Scientific, Inc.) were utilized to detect the expression of miRNAs, according to the manufacturer's protocol. U6 small nuclear RNA was used as an endogenous control.

\section{Western blotting.}

Primary antibodies of FGFR1 (cat. no. ab824; dilution, 1: 1000; Abcam, Cambridge, MA, USA) and $\beta$-actin (cat. no. sc-81178; dilution, 1: 2000; Santa Cruz Biotechnology, Inc., Dallas, TX, USA) were used to 
incubate membranes. The membranes were washed three times with TBST and incubated with secondary antibodies donkey anti-mouse IgG (sc-2096, dilution, 1:10,000; Santa Cruz Biotechnology, Inc.) for $2 \mathrm{~h}$ at room temperature and visualized with an Amersham ECL Western blotting detection reagent (GE Healthcare Life Sciences, Chalfont, UK).

\section{Immunohistochemical (IHC) staining}

The experimental procedures of IHC staining and the scoring rules of FGFR1-positive staining in HCC tissues and adjacent para-carcinoma tissues were referred to as previously described [12]. Primary antibody of FGFR1 (cat. no. ab824; dilution, 1: 100) was obtained from Abcam (Cambridge, UK).

\section{Statistical analysis.}

Data from these experiments were reported as mean \pm standard deviation for each group. All statistical analyses were performed using PRISM version 7.0 (GraphPad Software, Inc., La Jolla, CA, USA). The student $t$-test was used to analyze two-group differences. Inter-group differences were analyzed by oneway ANOVA. Differences with a $\mathrm{P}$ value of $<0.05$ were considered statistically significant.

\section{Results}

\section{ATL inhibited proliferation in HCC cells.}

To analyze the anti-proliferative activity of ATL on HCC cell lines (MHCC97H, HepG2, Huh7 and SMMC7721), cells were exposed to various concentrations of ATLfor 24,48 and 72 hours, and the CCK-8 assay was used to monitor the cell viability. The results demonstrated that the cell viability of $\mathrm{MHCC} 97 \mathrm{H}$ (Figure 1B), HepG2 (Figure 1C), Huh7 (Figure 1D) and SMMC7721 (Figure 1E) were inhibited by ATL treatment in a dose-dependent manner. We noticed that the cell viability of HepG2 (Figure 1C) and SMMC7721 (Figure 1E) was also inhibited by ATL treatment in a time-dependent pattern.

\section{ATL inhibited migration and induced apoptosis in HCC cell lines.}

The transwell assay was conducted to assess the migratory and migratory capability of HepG2 and SMMC7721 cells in the presence of ATL with different concentrations for 24 hours. As shown in Figure 
2A and Figure 2B, ATL had the ability to restrain migration and invasion in a dose-dependent manner in both the HepG2 and SMMC7721 cells. To study the anti-tumor activity of ATL, cell apoptosis was tested in ATL-treated HepG2 and SMMC7721 cells, which were stained with TUNEL, and then the proportion of TUNEL positive cells was measured by fluorescence microscope. As shown in Figure 2C, ATL induced cells apoptosis in a dose-dependent manner in both the HepG2 and SMMC7721 cells.

\section{ATL suppressed FGFR1 in HCC cells.}

To examine whether ATL-induced growth inhibition and apoptosis in HepG2 and SMMC7721 cells were due to regulate FGFR1 expression, the protein level was analyzed by western blotting. Our results demonstrated that FGFR1 was dramatically decreased in ATL-treated HepG2 and SMMC7721 cells in a dose-dependent manner (Figure 3A). To investigate its function in vitro, we constructed small interfering RNA (siRNA) vector targeting FGFR1, namely si-FGFR1. To assess the potential effects of siRNA-mediated FGFR1 silencing on cell proliferation, CCK-8 assay was performed at 24, 48 and 72 hours after si-FGFR1 or siRNA control (si-NC) transfection. Compared with the si-NC group, a significant decrease in cell viability was detected in HepG2 and SMMC7721 cells after transfected with si-FGFR1 (Figure 3A). We further analyzed apoptosis using flow cytometry in HepG2 and SMMC7721 cells after transfected with siFGFR1 or si-NC (Figure 3B). Compared with the si-NC group, si-FGFR1 transfected induced cells apoptosis in both the HepG2 and SMMC7721 cells for 48 hours after transfection (Figure 3C). Furthermore, we analyzed migration and invasion in HepG2 and SMMC7721 cells after transfected with si-FGFR1 or si-NC for 24 hours. The migration and invasion were significantly inhibited in HepG2 and SMMC7721 cells after transfected with si-FGFR1 (Figure 3D and 3E). Thus, our present results indicate that FGFR1 may facilitate HCC malignant progression, inhibition of FGFR1 can inhibit proliferation, migration and invasion, as well as induce apoptosis in HCC cells.

\section{FGFR1 was a downstream target of miR-195-5p.}

To further investigate whether ATL modulated FGFR1 expression via a post-transcriptional regulatory mechanism, a miRNA integrated-signature of 13 miRNAs (miR-93-5p, miR-224-5p, miR-222-3p, miR-2213p, miR-21-5p, miR-223-3p, miR-214-3p, miR-199a-5p, miR-199a-3p, miR-195-5p, miR-150-5p, miR-145-5p and miR-130a-3p), including 5 upregulated and 8 downregulated, from 26 published datasets in HCC was included in our study [22]. Using bioinformatics algorithms, we revealed that 7 miRNAs, including miR-935p, miR-214-3p, miR-199a-5p, miR-195-5p, miR-150-5p, miR-145-5p and miR-130a-3p, had the complementary sequence in the 3'-UTR of FGFR1 mRNA. Intriguingly, ATL treatment significantly upregulated miR-199a-5p, miR-195-5p and miR-130a-3p and down-regulated miR-93-5p expression compared with control group (Figure 4A). The expression level of miR-195-5p was higher than other 
miRNAs after ATL treatment. Therefore, we focused on miR-195-5p in further study. As shown in Figure 4B, the conserved complementary pairing sequences between miR-195-5p and the 3'-UTR of FGFR1 were predicted using TargetScanHuman 7.2 (http://www.targetscan.org/). To confirm this prediction, we performed luciferase reporter assay, and the results showed that transfection of miR-195-5p mimics significantly reduced the luciferase activity in HepG2 and SMMC7721 cells containing WT 3'-UTR of FGFR1, while the luciferase activity had no obvious change in HepG2 and SMMC7721 cells containing Mut 3'-UTR of FGFR1 (Figure 4C). We also found that the down-regulation of FGFR1 protein expression was observed in HepG2 and SMMC7721 cells after transfection with miR-195-5p mimics (Figure 4D).

\section{miR-195-5p expression is negatively correlated with FGFR1 in HCC tissues.}

As shown in Figure 5A, miR-195-5p expression was significantly decreased in HCC tissues compared with corresponding para-carcinoma tissues. HCC patients with high miR-195-5p expression showed a longer overall survival than those of patients with low miR-195-5p expression (Figure 5B). IHC staining indicated that FGFR1 protein expression exhibited a significant elevation in HCC tissues compared with corresponding para-carcinoma tissues (Figure 5C and 5D). HCC patients with high FGFR1 expression showed a shorter overall survival than those of patients with low FGFR1 expression (Figure 5E). Correlation analysis suggested that miR-195-5p expression is negatively correlated with FGFR1 in HCC tissues (Figure 5F).

\section{miR-195-5p-caused growth inhibition and apoptosis were reversed by overexpressed FGFR1.}

miR-195-5p mimics and FGFR1 overexpressed plasmids were co-transfected into HepG2 and SMMC7721 cells. Overexpression of miR-195-5p significantly suppressed proliferation (Figure 6A) and induced apoptosis (Figure 6B and 6C), as well as blocked migration and invasion (Figure 6D) in HepG2 and SMMC7721 cells. However, the tumor-suppressive functions of miR-195-5p were neutralized by overexpressed FGFR1 in HepG2 and SMMC7721 cells (Figure 6A-6D). These findings suggest that miR195-5p and FGFR1 may play reciprocal roles in the carcinogenesis of HCC. These results suggest that ATL might be a potential therapeutic drug for HCC, and the underlying mechanism was mediated, at least partially, through the up-regulation of miR-195-5p to repress FGFR1 expression (Figure 7).

\section{Discussion}


The present study investigated the anti-proliferative and pro-apoptotic effects of ATL in HCC cells. When ATL was exposed to the HepG2 and SMMC7721 cells, the growth, migration and invasion of these cell lines were dramatically inhibited. Moreover, ATL treatment resulted in a significant increase in cell apoptosis of HCC cells. Intriguingly, we observed that ATL unexpectedly inhibited FGFR1 in HCC cells. The knockdown of FGFR1 also inhibited proliferation and induced apoptosis in HCC cells. In addition, we discovered that ATL treatment led to the up-regulation of miR-195-5p expression, which as a posttranscriptional targeted FGFR1. The anti-proliferative and pro-apoptotic roles of miR-195-5p were neutralized by overexpressed FGFR1 in HCC cells.

The activation of the apoptotic pathway in cancer cells can serve as a defensive mechanism against the proliferation of cancer cells [26]. Pro-apoptotic protein, BAX, and anti-apoptotic protein, Bcl-2, are closely related to mitochondrial apoptosis by enhancing mitochondrial membrane permeabilization and activating caspase-3 expression [27]. First, we detected the effects of ATL on cell apoptosis by TUNEL staining, which showed that the percentage of apoptotic cells was significantly increased and positively related to the concentrations of ATL in HCC cells. Taken together, these findings indicate that ATL induces apoptosis in human HCC cells and may be a potential drug for cancer therapy.

Mounting evidence has shown that FGFR signaling plays crucial roles in cancer cell proliferation, migration, angiogenesis and survival by targeting mitogen-activated protein kinase (MAPK) signaling pathway, including ERK, p38 MAPK and c-Jun N-terminal kinase (JNK) pathways [12, 28]. Fibroblast growth receptor family includes FGFR1, 2, 3 and 4 that serve as receptor tyrosine kinases [29]. FGFR1 activation has been found in a number of human cancers and plays a crucial role in drug resistance, cancer progression and metastasis $[19,30,31]$. Wang et al have been suggested that FGFR1 can be regulated by non-coding RNA and can serve as a potential therapeutic target for HCC [12]. To explain the possible mechanism underlying ATL-induced HCC cells apoptosis, we examined the protein expression levels of FGFR1 in HCC cells. Strikingly, FGFR1 was significantly inhibited in ATL-treated HCC cells. Inhibition of FGFR1 showed a significant growth inhibition and apoptosis in HCC cells. Our results suggest that ALT may possess the anti-cancer capability that provides a new potential therapeutic drug for HCC treatment.

We also discovered that ALT treatment increased the expression of miR-195-5p in HepG2 and SMMC7721 cells. Based on this conclusion, we attempted to explore the roles of miR-195-5p in the progression of HCC. In vitro experiments validated that over-expression of miR-195-5p inhibited proliferation and promoted apoptosis in HCC cells, as well as reduced the protein expression of FGFR1. Interestingly, the antineoplastic activities of miR-195-5p were counteracted by overexpressed FGFR1 in vitro. Shi et al demonstrate that the expression of miR-195-5p is decreased in the HCC tissues, which was also validated 
on the TCGA dataset [22]. Zhu et al also exhibit that miR-195-5p is lower in the HCC tissues than in the matched non-tumor tissues [32]. Xu et al indicate that miR-195-5p acts as an anti-oncogene in HCC, reflecting that overexpression of miR-195-5p leads to suppression of invasion, migration and proliferation in vitro [33]. Consistent with these results, we also found that overexpression of miR-195-5p showed an anti-proliferative and pro-apoptotic activities by inhibiting FGFR1 expression in HCC cells.

In summary, our study has shown the potential of ATL for the efficient induction of human HCC cells growth inhibition and apoptosis, and the underlying mechanism is mediated, at least partially, via targeting miR-195-5p/FGFR1 signaling pathway. Consequently, we will further study the chemosensitization strategies of ATL in vivo to gain insights to aid in the development of an effective strategy for reversing the drug resistance of HCC.

\section{Abbreviations}

ATL, atractylenolide III; HCC, hepatocellular carcinoma; FGFR1, fibroblast growth factor receptor1; ERK, extracellular signal regulated kinase; miRNA or miR, microRNA; 3'-UTR, 3'-untranslated region; mRNA, messenger RNA; siRNA, small interfering RNA.

\section{Declarations}

\section{Ethics approval and consent to participate:}

The clinical research was approved by the Ethics Committee of the Xiangya Hospital of Central South University. Informed consent forms were approved by all of the patients.

\section{Consent for publication:}

Not applicable.

\section{Availability of data and material:}

The data that support the findings of this study are available from the corresponding author upon reasonable request.

\section{Competing interests:}

The authors declare they have no competing interests. 


\section{Funding:}

Not applicable.

\section{Authors' contributions:}

Study design: $L S$; Literature research, Data acquisition and Data analysis: $L S, J L, N L, L G, L L, Q Z, X L, H L$ and ZC; Manuscript preparation and Manuscript editing: LS, JL, NL, LG, LL, QZ, XL, HL and ZC; Manuscript review: LS; Cell experiments: LS, JL, NL, LG, LL, QZ, XL, HL and ZC. All authors read and approved the final manuscript.

\section{Acknowledgements:}

Not applicable.

\section{References}

1. Jo JC, Choi EK, Shin JS, Moon JH, Hong SW, Lee HR, Kim SM, Jung SA, Lee DH, Jung SH, et al: Targeting FGFR Pathway in Human Hepatocellular Carcinoma: Expressing pFGFR and pMET for Antitumor Activity. Mol Cancer Ther 2015, 14:2613-2622.

2. Colagrande S, Inghilesi AL, Aburas S, Taliani GG, Nardi C, Marra F: Challenges of advanced hepatocellular carcinoma. World J Gastroenterol 2016, 22:7645-7659.

3. Dekervel J, van Malenstein H, Vandecaveye V, Nevens F, van Pelt J, Heye S, Laleman W, Van Steenbergen W, Vaninbroukx J, Verslype C, Maleux G: Transcatheter arterial chemoembolization with doxorubicin-eluting superabsorbent polymer microspheres in the treatment of hepatocellular carcinoma: midterm follow-up. J Vasc Interv Radiol 2014, 25:248-255.e241.

4. Gruber-Rouh T, Schmitt C, Naguib NNN, Nour-Eldin NA, Eichler K, Beeres M, Vogl TJ: Transarterial chemoembolization (TACE) using mitomycin and lipiodol with or without degradable starch microspheres for hepatocellular carcinoma: comparative study. BMC Cancer 2018, 18:188.

5. Nakano M, Niizeki T, Nagamatsu H, Tanaka M, Kuromatsu R, Satani M, Okamura S, Iwamoto H, Shimose S, Shirono T, et al: Clinical effects and safety of intra-arterial infusion therapy of cisplatin suspension in lipiodol combined with 5-fluorouracil versus sorafenib, for advanced hepatocellular carcinoma with macroscopic vascular invasion without extra-hepatic spread: A prospective cohort study. Mol Clin Oncol 2017, 7:1013-1020.

6. Cox J, Weinman S: Mechanisms of doxorubicin resistance in hepatocellular carcinoma. Hepat Oncol 2016, 3:57-59.

7. Kim Y, Jang M, Lim S, Won H, Yoon KS, Park JH, Kim HJ, Kim BH, Park WS, Ha J, Kim SS: Role of cyclophilin $\mathrm{B}$ in tumorigenesis and cisplatin resistance in hepatocellular carcinoma in humans. Hepatology 2011, 54:1661-1678. 
8. Liu C, Zhao H, Ji ZH, Yu XY: Neuroprotection of atractylenolide III from Atractylodis macrocephalae against glutamate-induced neuronal apoptosis via inhibiting caspase signaling pathway. Neurochem Res 2014, 39:1753-1758.

9. Kang TH, Bang JY, Kim MH, Kang IC, Kim HM, Jeong HJ: Atractylenolide III, a sesquiterpenoid, induces apoptosis in human lung carcinoma A549 cells via mitochondria-mediated death pathway. Food Chem Toxicol 2011, 49:514-519.

10. Li CQ, He LC, Jin JQ: Atractylenolide I and atractylenolide III inhibit Lipopolysaccharide-induced TNFalpha and NO production in macrophages. Phytother Res 2007, 21:347-353.

11. Ji GQ, Chen RQ, Wang L: Anti-inflammatory activity of atractylenolide III through inhibition of nuclear factor-kappaB and mitogen-activated protein kinase pathways in mouse macrophages. Immunopharmacol Immunotoxicol 2016, 38:98-102.

12. Wang F, Ying HQ, He BS, Pan YQ, Deng QW, Sun HL, Chen J, Liu X, Wang SK: Upregulated IncRNAUCA1 contributes to progression of hepatocellular carcinoma through inhibition of miR-216b and activation of FGFR1/ERK signaling pathway. Oncotarget 2015, 6:7899-7917.

13. Belo A, Cheng K, Chahdi A, Shant J, Xie G, Khurana S, Raufman JP: Muscarinic receptor agonists stimulate human colon cancer cell migration and invasion. Am J Physiol Gastrointest Liver Physiol 2011, 300:G749-760.

14. Ji W, Yu Y, Li Z, Wang G, Li F, Xia W, Lu S: FGFR1 promotes the stem cell-like phenotype of FGFR1amplified non-small cell lung cancer cells through the Hedgehog pathway. Oncotarget 2016, 7:1511815134.

15. Cheng CL, Thike AA, Tan SY, Chua PJ, Bay BH, Tan PH: Expression of FGFR1 is an independent prognostic factor in triple-negative breast cancer. Breast Cancer Res Treat 2015, 151:99-111.

16. Theelen WS, Mittempergher L, Willems SM, Bosma AJ, Peters DD, van der Noort V, Japenga EJ, Peeters T, Koole K, Sustic T, et al: FGFR1, 2 and 3 protein overexpression and molecular aberrations of FGFR3 in early stage non-small cell lung cancer. J Pathol Clin Res 2016, 2:223-233.

17. Murase H, Inokuchi M, Takagi Y, Kato K, Kojima K, Sugihara K: Prognostic significance of the cooverexpression of fibroblast growth factor receptors 1, 2 and 4 in gastric cancer. Mol Clin Oncol 2014, 2:509-517.

18. Wen D, Li S, Ji F, Cao H, Jiang W, Zhu J, Fang X: miR-133b acts as a tumor suppressor and negatively regulates FGFR1 in gastric cancer. Tumour Bio/ 2013, 34:793-803.

19. Riehle KJ, Yeh MM, Yu JJ, Kenerson HL, Harris WP, Park JO, Yeung RS: mTORC1 and FGFR1 signaling in fibrolamellar hepatocellular carcinoma. Mod Pathol 2015, 28:103-110.

20. Xie Q, Yang Y, Wang Z, Chen F, Zhang A, Liu C: Resveratrol-4-O-D-(2'-galloyl)-glucopyranoside isolated from Polygonum cuspidatum exhibits anti-hepatocellular carcinoma viability by inducing apoptosis via the JNK and ERK pathway. Molecules 2014, 19:1592-1602.

21. Croce CM: Causes and consequences of microRNA dysregulation in cancer. Nat Rev Genet 2009, 10:704-714. 
22. Shi KQ, Lin Z, Chen XJ, Song M, Wang YQ, Cai YJ, Yang NB, Zheng MH, Dong JZ, Zhang L, Chen YP: Hepatocellular carcinoma associated microRNA expression signature: integrated bioinformatics analysis, experimental validation and clinical significance. Oncotarget 2015, 6:25093-25108.

23. Wu J, Ji A, Wang X, Zhu Y, Yu Y, Lin Y, Liu Y, Li S, Liang Z, Xu X, et al: MicroRNA-195-5p, a new regulator of Fra-1, suppresses the migration and invasion of prostate cancer cells. $J$ Trans/ Med 2015, 13:289.

24. Fei X, Qi M, Wu B, Song Y, Wang Y, Li T: MicroRNA-195-5p suppresses glucose uptake and proliferation of human bladder cancer T24 cells by regulating GLUT3 expression. FEBS Lett 2012, 586:392-397.

25. Yang R, Xing L, Zheng X, Sun Y, Wang X, Chen J: The circRNA circAGFG1 acts as a sponge of miR$195-5 p$ to promote triple-negative breast cancer progression through regulating CCNE1 expression. 2019, 18:4.

26. Yu R, Yu BX, Chen JF, Lv XY, Yan ZJ, Cheng Y, Ma Q: Anti-tumor effects of Atractylenolide I on bladder cancer cells. J Exp Clin Cancer Res 2016, 35:40.

27. Wong RS: Apoptosis in cancer: from pathogenesis to treatment. J Exp Clin Cancer Res 2011, 30:87.

28. Ahmad I, Iwata T, Leung HY: Mechanisms of FGFR-mediated carcinogenesis. Biochim Biophys Acta 2012, 1823:850-860.

29. Touat M, lleana E, Postel-Vinay S, Andre F, Soria JC: Targeting FGFR Signaling in Cancer. Clin Cancer Res 2015, 21:2684-2694.

30. Azuma K, Kawahara A, Sonoda K, Nakashima K, Tashiro K, Watari K, Izumi H, Kage M, Kuwano M, Ono $M$, Hoshino T: FGFR1 activation is an escape mechanism in human lung cancer cells resistant to afatinib, a pan-EGFR family kinase inhibitor. Oncotarget 2014, 5:5908-5919.

31. Yang F, Zhang Y, Ressler SJ, Ittmann MM, Ayala GE, Dang TD, Wang F, Rowley DR: FGFR1 is essential for prostate cancer progression and metastasis. Cancer Res 2013, 73:3716-3724.

32. Zhu HR, Huang RZ, Yu XN, Shi X, Bilegsaikhan E, Guo HY, Song GQ, Weng SQ, Dong L, Janssen HLA, et al: Microarray Expression Profiling of microRNAs Reveals Potential Biomarkers for Hepatocellular Carcinoma. Tohoku J Exp Med 2018, 245:89-98.

33. Xu H, Hu YW, Zhao JY, Hu XM, Li SF, Wang YC, Gao JJ, Sha YH, Kang CM, Lin L, et al: MicroRNA-195$5 p$ acts as an anti-oncogene by targeting PHF19 in hepatocellular carcinoma. Oncol Rep 2015, $34: 175-182$.

\section{Figures}


A

Atractylenolide III<smiles>C=C1CCC[C@]2(C)C[C@]3(O)OC(=O)C(C)=C3C[C@H]12</smiles>

Molecular formula: $\mathrm{C}_{15} \mathrm{H}_{20} \mathrm{O}_{3}$
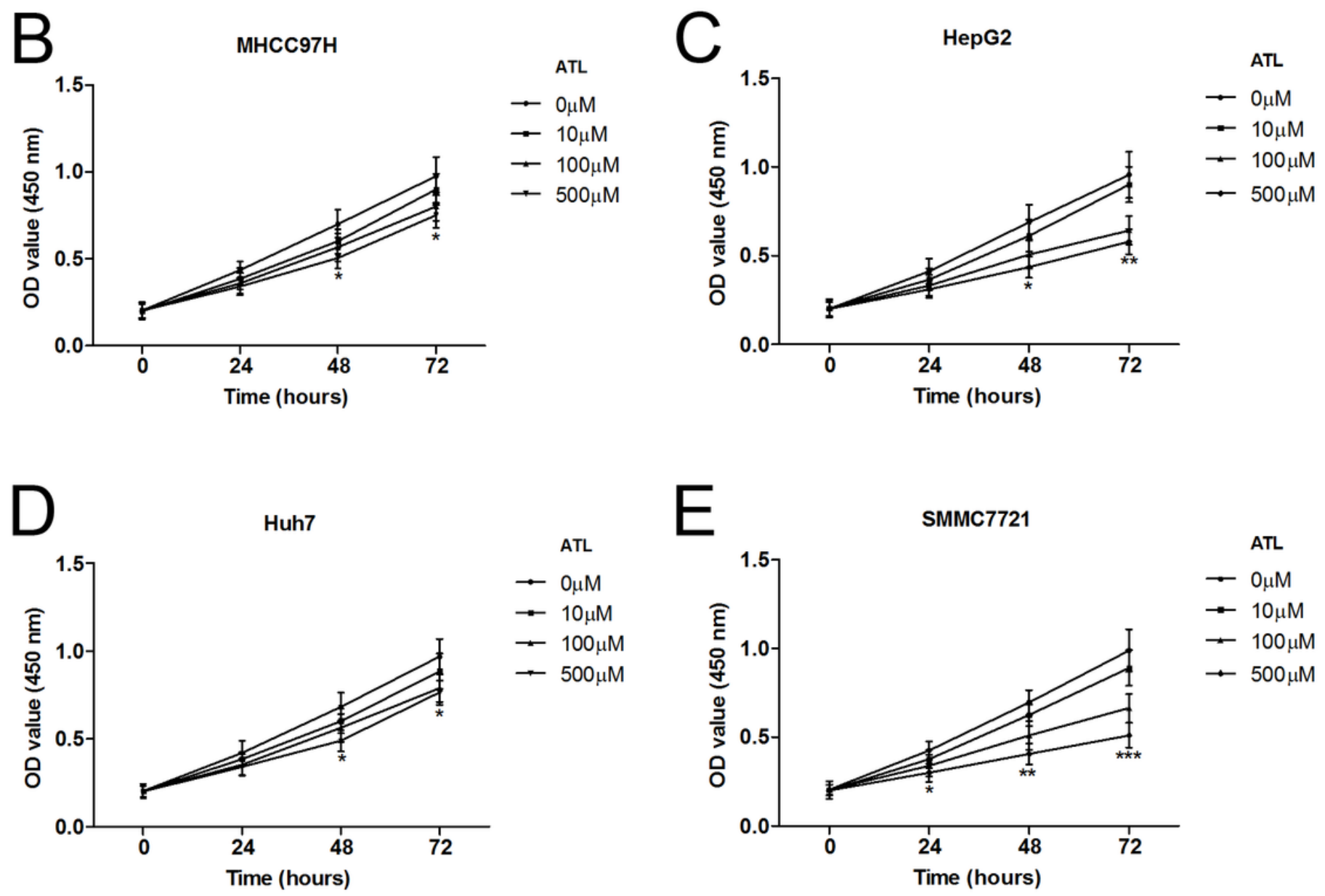

Figure 1

ATL inhibited proliferation in HCC cells. Molecular structure of Atractylenolide III (Molecular weight: 248.32; A). MHCC97H (B), HepG2 (C), Huh7 (D) and SMMC7721 (E) cells viability were detected by CCK-8 assay in the presence of ATL. $n=3$ in each group. ${ }^{*} \mathrm{P}<0.05$, $* * \mathrm{P}<0.01$ and $* \star * \mathrm{P}<0.001$ compared with control group. 
A
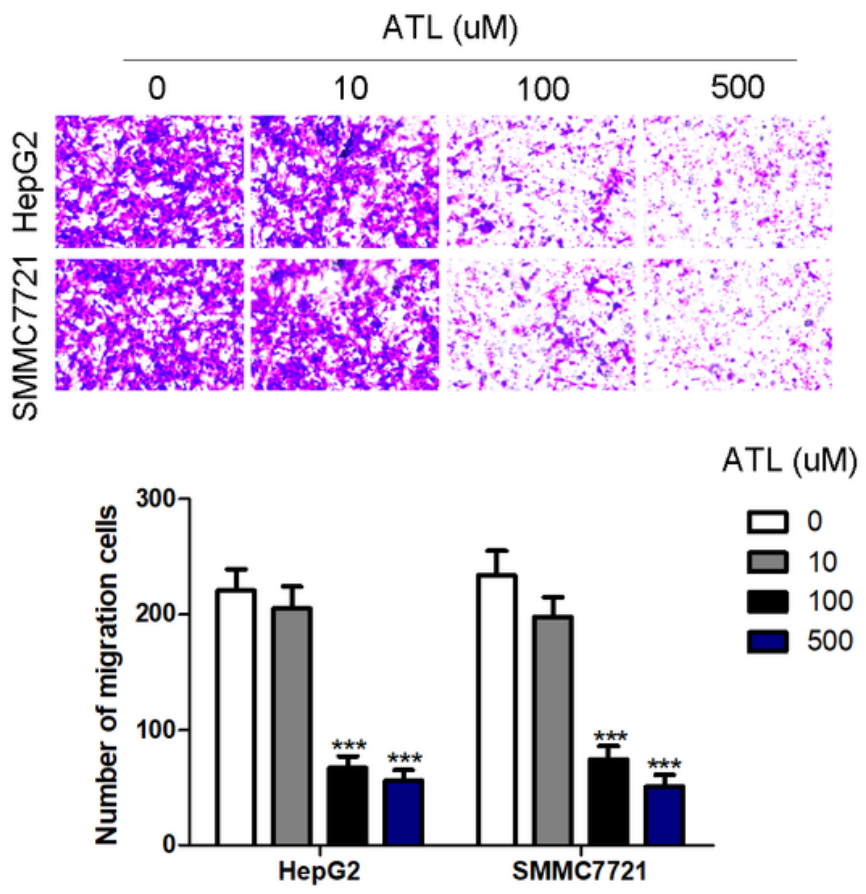

B

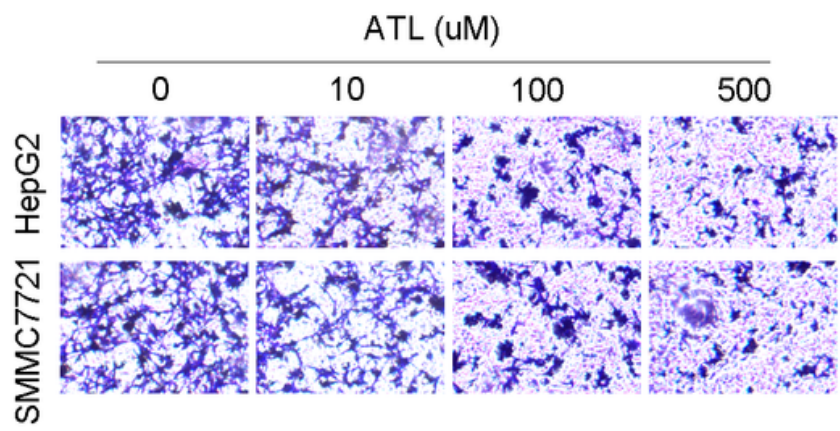

ATL (UM)

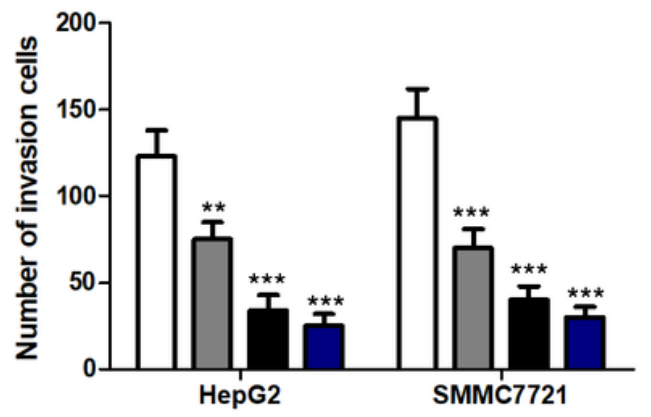

C
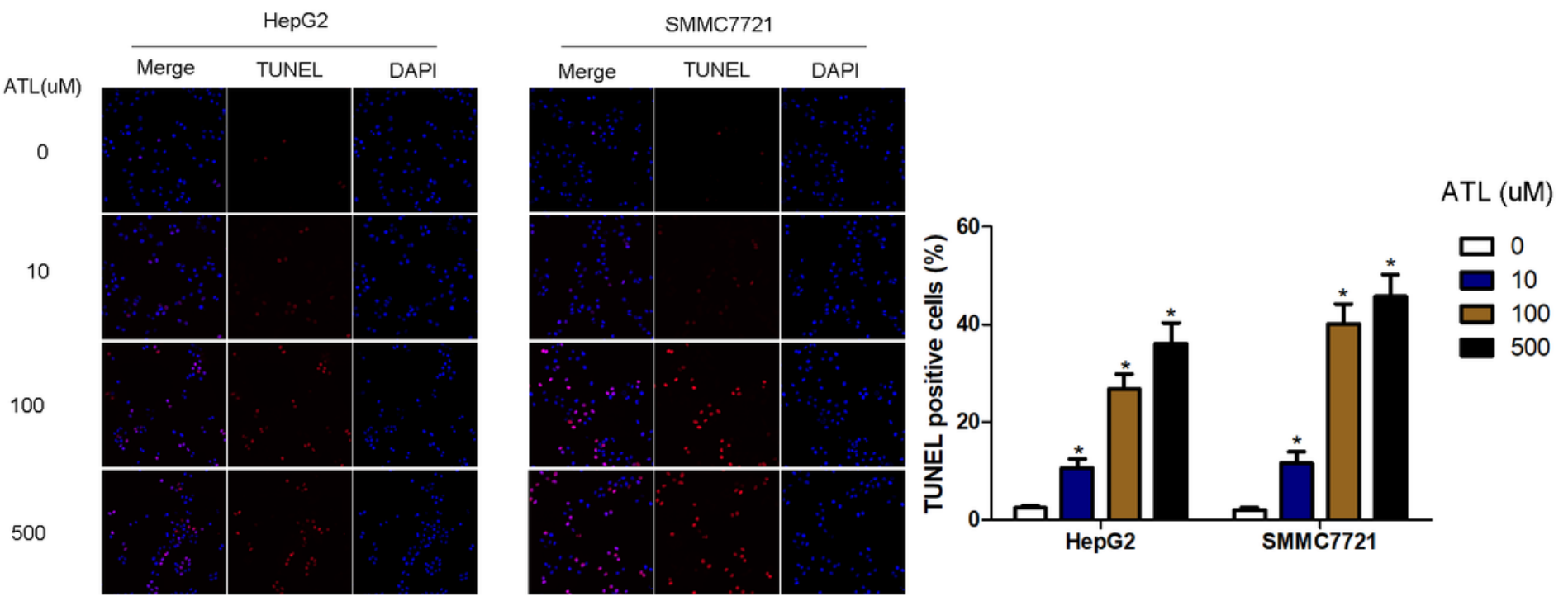

Figure 2

ATL inhibited migration and invasion in HCC cell lines. The transwell assay was conducted to assess migration and invasion of HepG2 (A) and SMMC7721 (B) cells in the presence of ATL with different concentrations for 24 hours. Cell apoptosis was evaluated using TUNEL staining in HepG2 and SMMC7721 cells with ATL treatment for 48 hours $(C)$. $n=3$ in each group. * $P<0.05, * \star P<0.01$ and $* \star * P$ $<0.001$ compared with control group. 

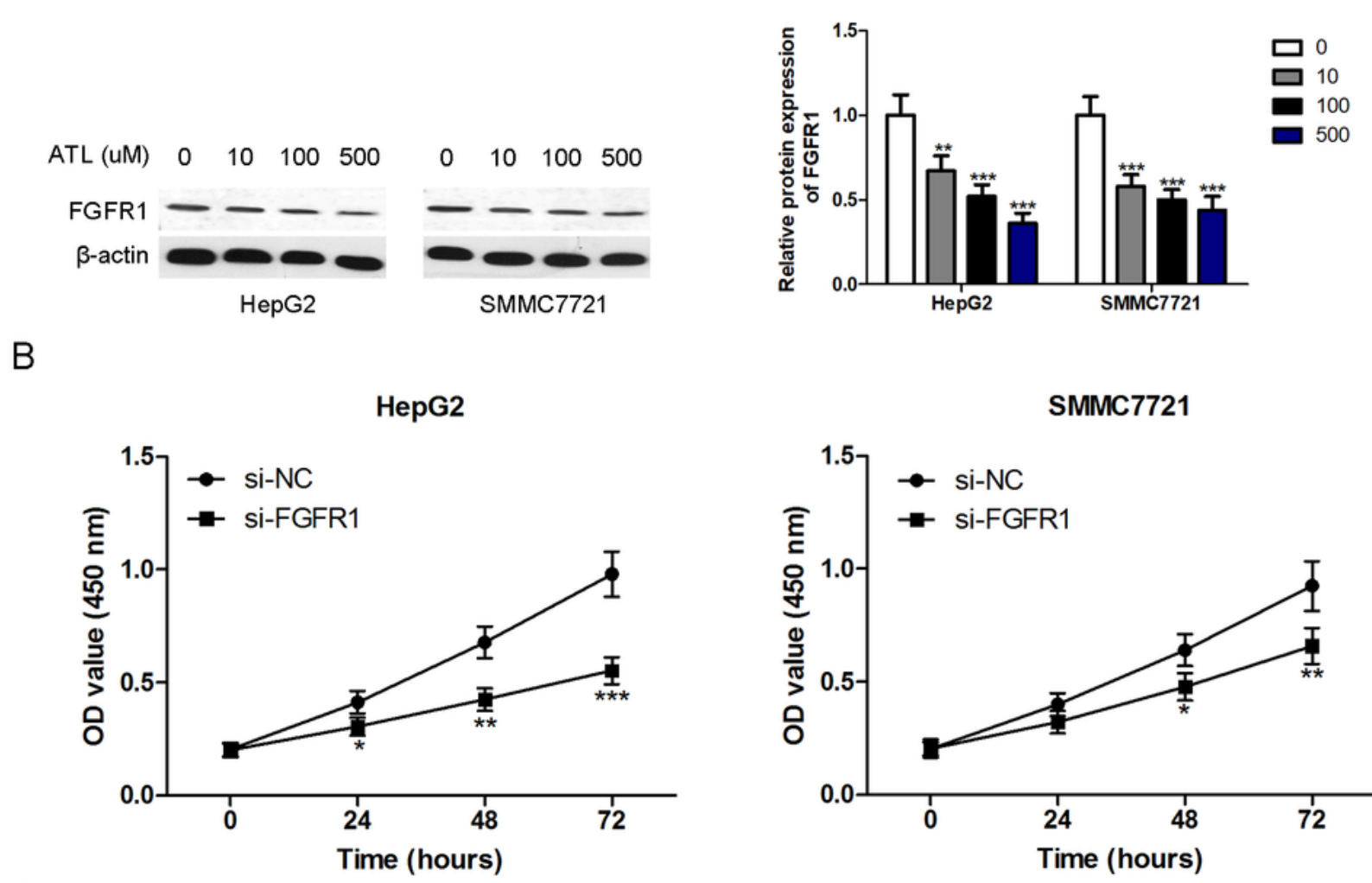

C

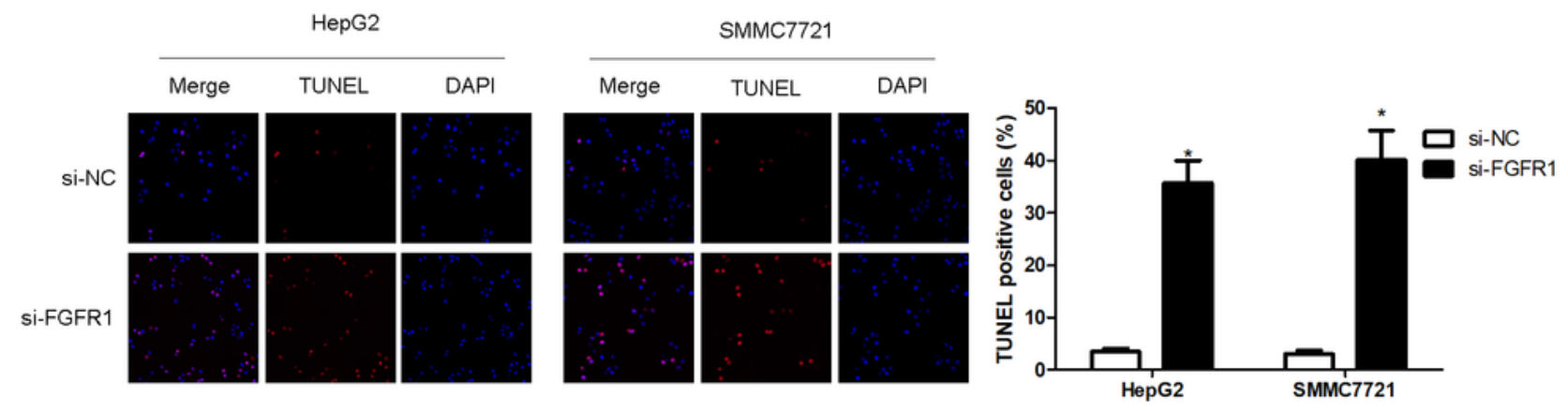

D

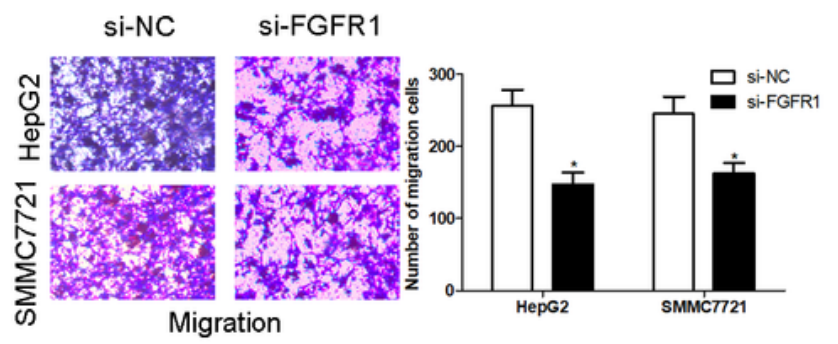

E

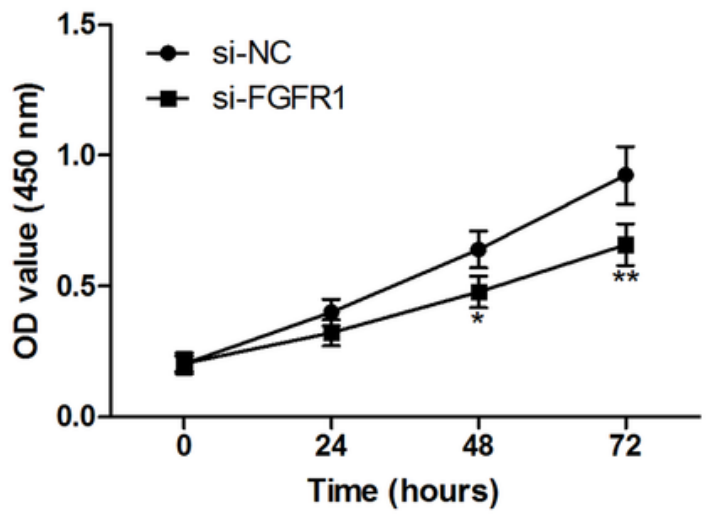


migration and invasion in HepG2 and SMMC7721 cells ( $D$ and E). $n=3$ in each group. ${ }^{\star} P<0.05, * \star P<$ 0.01 and $* \star * \mathrm{P}<0.001$ compared with control group.

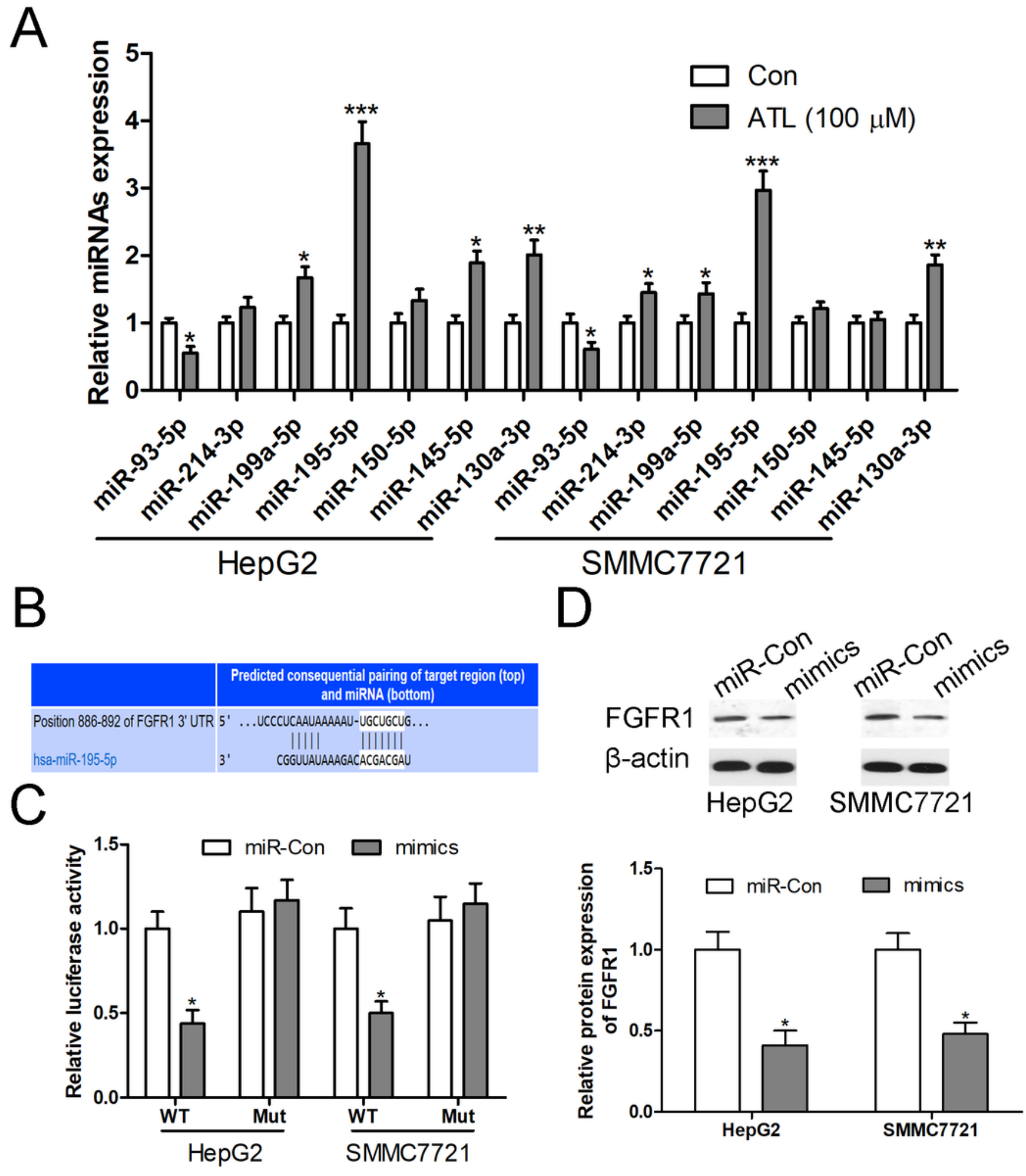

Figure 4

FGFR1 was a downstream target of miR-195-5p. The expression levels of miRNAs were measured by RTqPCR in HepG2 and SMMC7721 cells with or without ATL treatment (A). Complementary pairing sequences between miR-195-5p and FGFR1 were predicted using TargetScan (B). Luciferase reporter 
assay was performed to verify FGFR1 as a direct target of FGFR1 (C). After transfection with miR-Con or miR-195-5p mimics into HepG2 and SMMC7721 cells, the protein expression of FGFR1 was detected using western blotting (D). * $P<0.05$, ** $P<0.01$ and $* \star \star ~ P<0.001$ compared with corresponding control group.

A

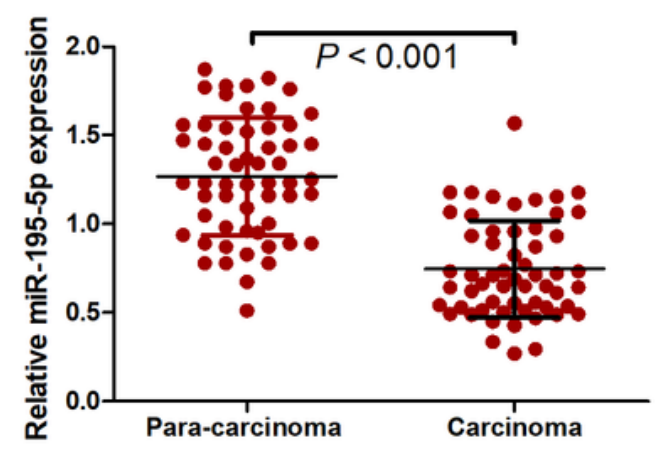

C
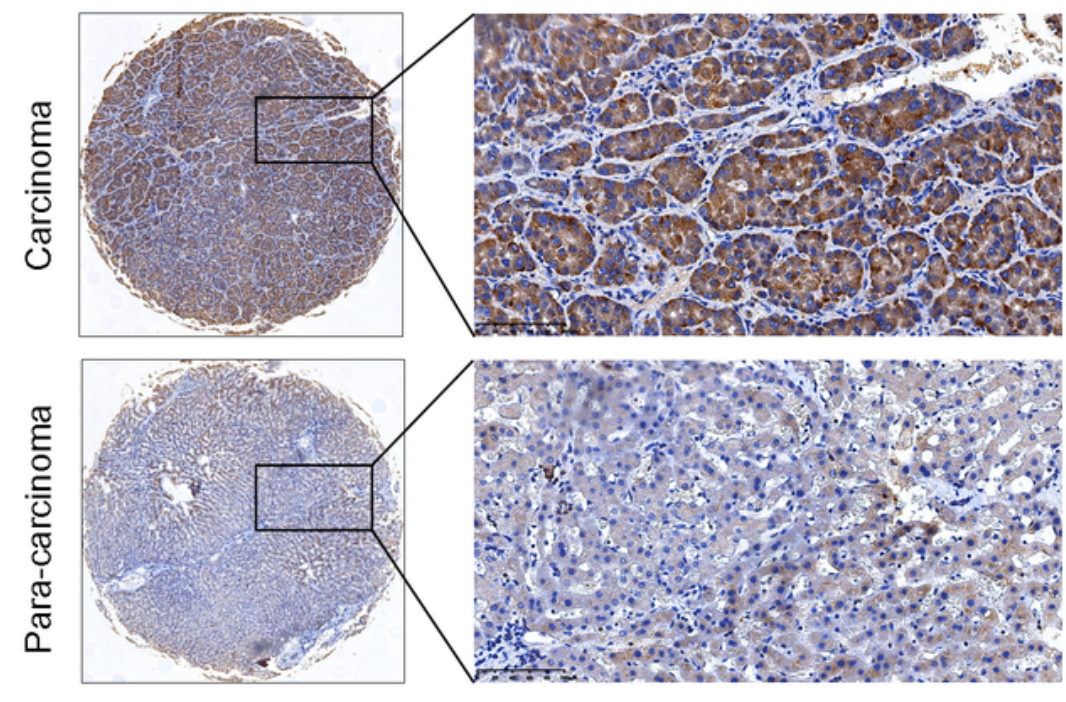

E

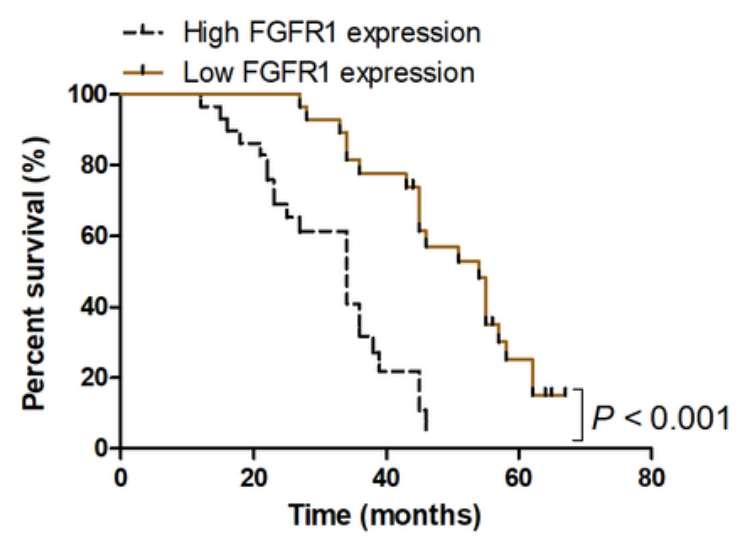

F
B

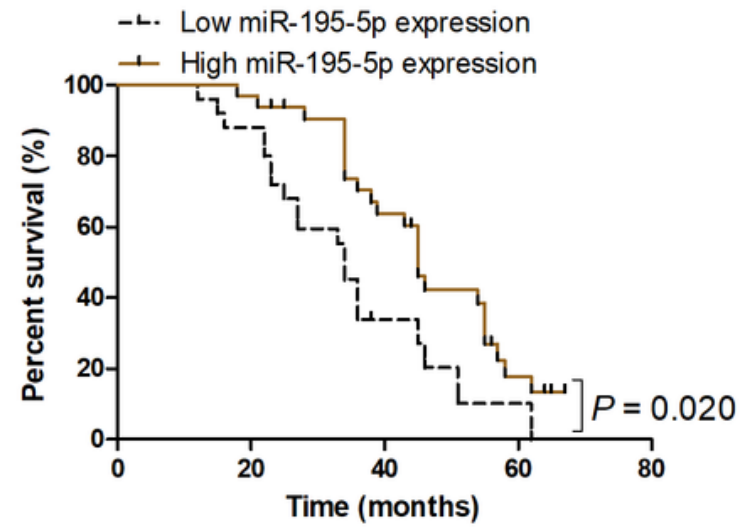

D
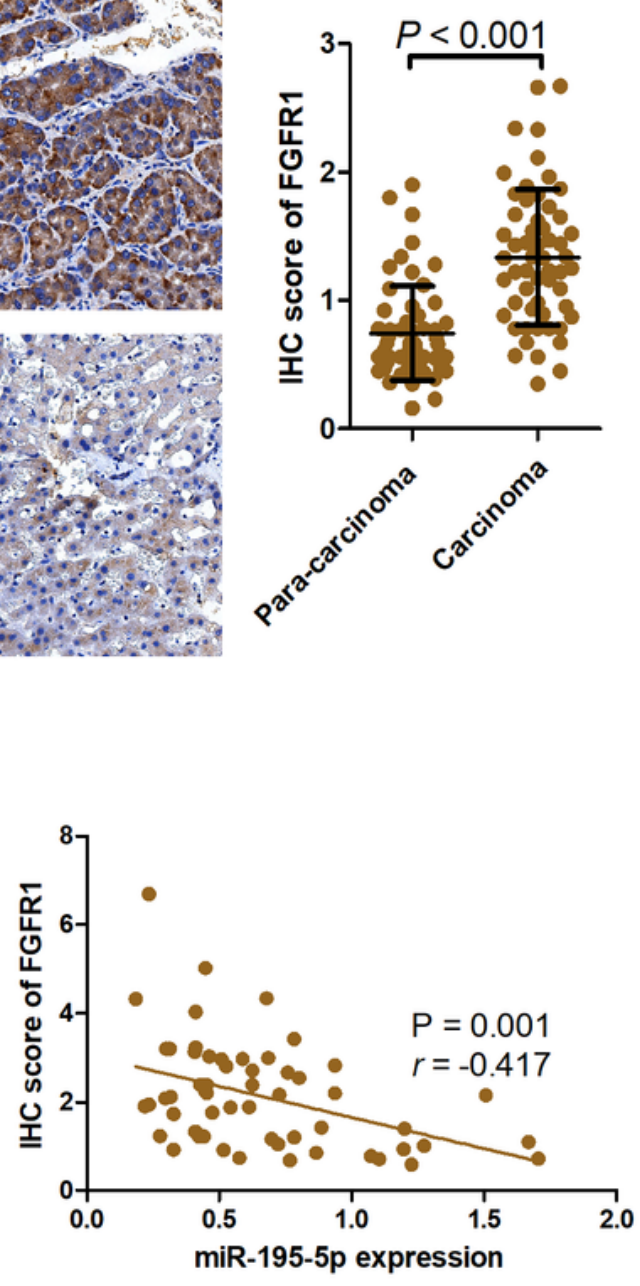

Figure 5 
miR-195-5p expression was negatively correlated with FGFR1 in HCC tissues. miR-195-5p expression in fifth-seven pairs of HCC tissues and corresponding para-carcinoma tissues was measured using RT-qPCR (A). HCC patients with high miR-195-5p expression showed a longer overall survival than those of patients with low miR-195-5p expression (B). IHC staining was used to evaluated FGFR1 protein in HCC tissues and corresponding para-carcinoma tissues (C and D). HCC patients with high FGFR1 expression showed a shorter overall survival than those of patients with low FGFR1 expression (E). Pearson correlation analysis was used to analyze the correlation between miR-195-5p and FGFR1 in HCC tissues (F).

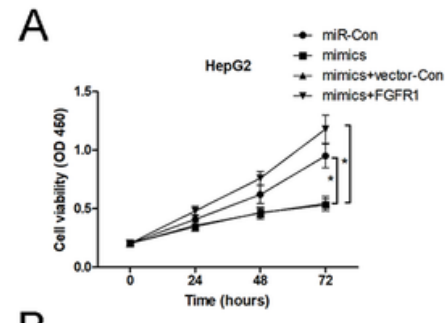

B

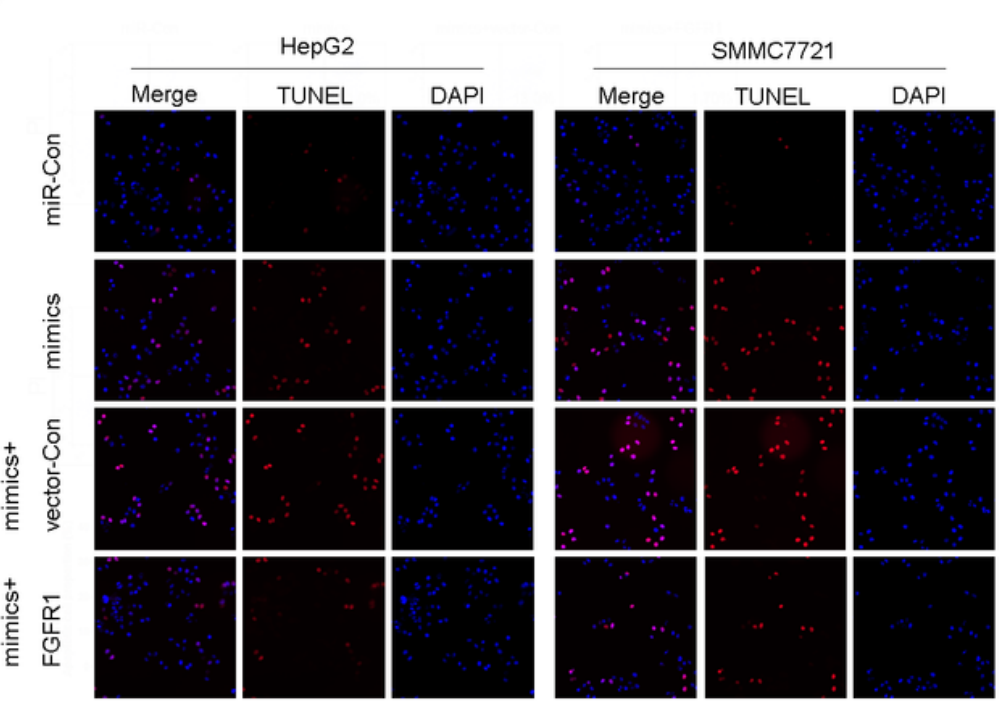

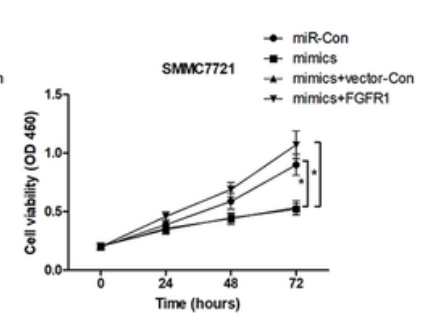

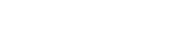

(2)
D
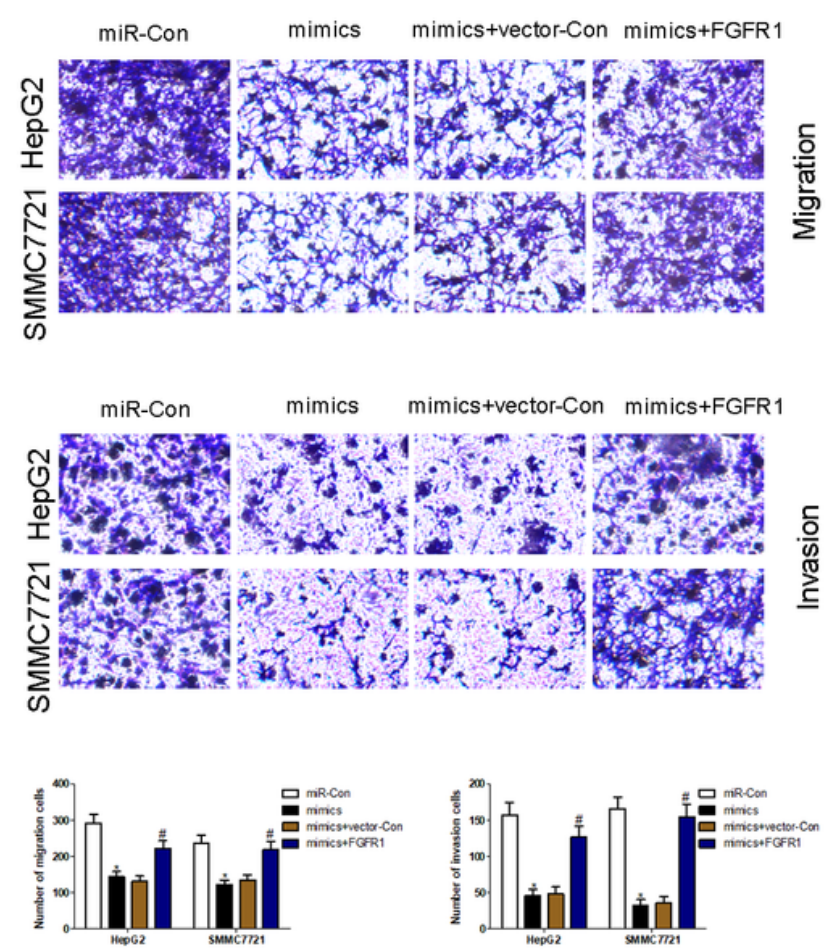

C

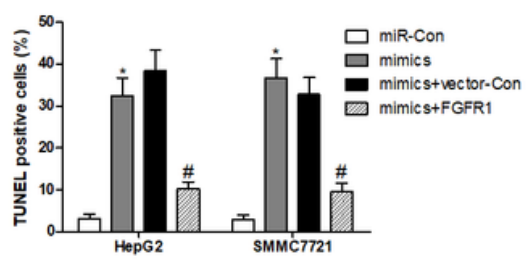

\section{Figure 6}

miR-195-5p-caused growth inhibition and apoptosis were reversed by overexpressed FGFR1. After cotransfection with miR-195-5p mimics and FGFR1 overexpressed plasmids into HepG2 and SMMC7721 cells, cell viability was detected by CCK-8 assay (A); cell apoptosis was measured using TUNEL staining ( $B$ and $C$ ); the transwell assay was conducted to assess migration and invasion (D). * $P<0.05$ compared with corresponding control group; \# $\mathrm{P}<0.05$ compared with mimics + vector-Con group. 


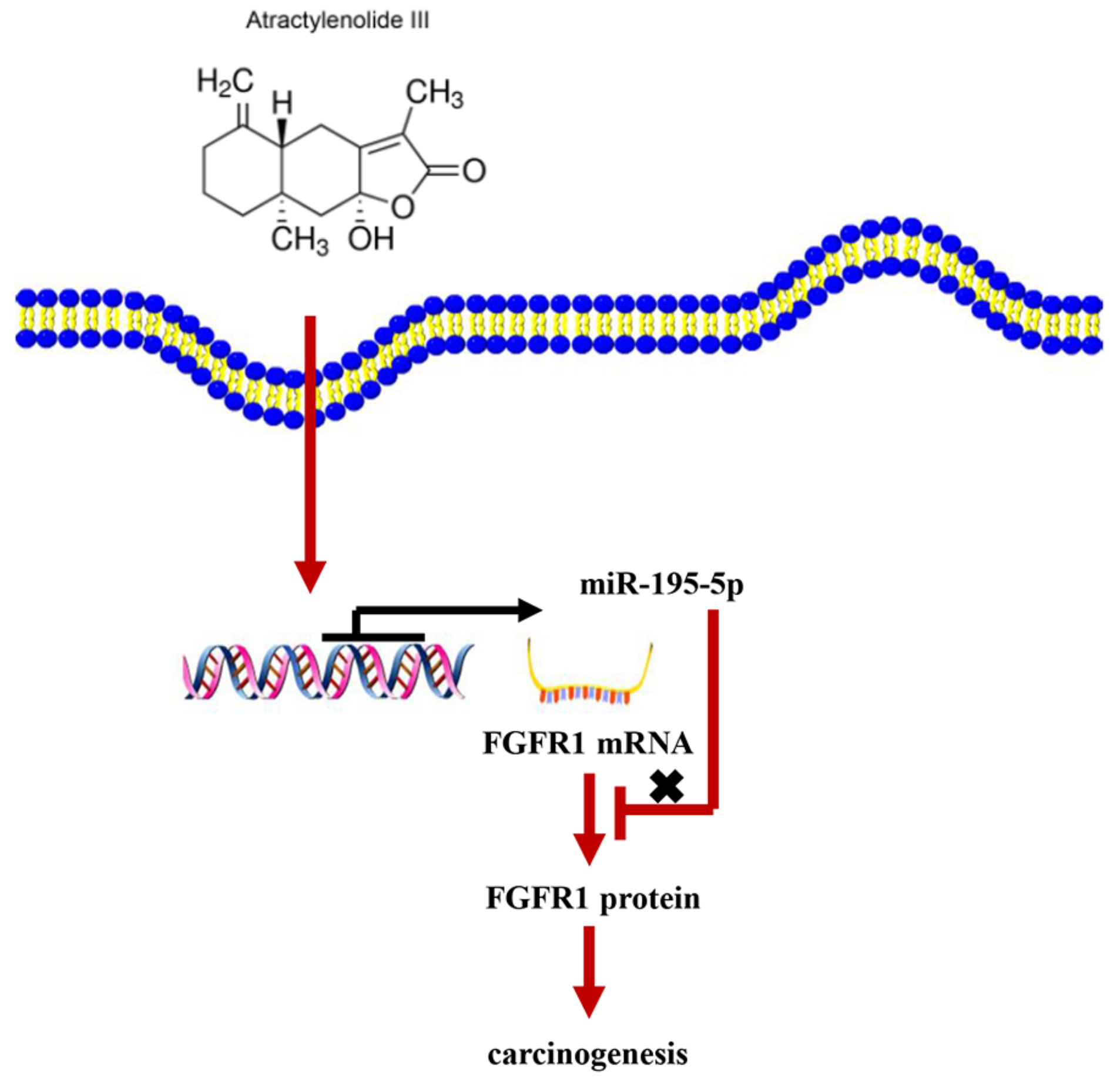

Figure 7

A schematic diagram for the effect of atractylenolide III to exert tumor-suppressive functions in liver cancer by targeting miR-195-5p/FGFR1 signaling axis. 